\title{
Stage IV Penile Cancer AJCC v6
}

National Cancer Institute

\section{Source}

National Cancer Institute. Stage IV Penile Cancer A/CC v6. NCI Thesaurus. Code C8959.

Stage IV includes: (T4, Any N, M0); (Any T, N3, M0); (Any T, Any N, M1). T4: Tumor

invades other adjacent structures. N3: Metastasis in deep inguinal or pelvic lymph node(s)

unilateral or bilateral. M1: Distant metastasis. (AJCC 6th ed.) - 2003 